\title{
Hemorragia de vías digestivas altas por presentación atípica de adenocarcinoma endometrial metastásico en el estómago. Tipificación diagnóstica con recursos endoscópicos
}

\section{Upper gastrointestinal bleeding due to atypical presentation of metastatic endometrial adenocarcinoma in the stomach. Diagnostic typing with endoscopic resources}

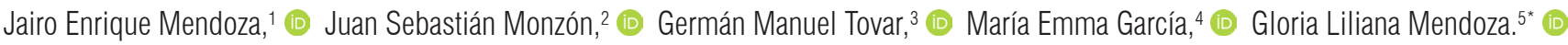

\author{
Gacceso abierto \\ Citación: \\ Mendoza JE, Monzón JS, Tovar GM, García \\ ME, Mendoza GL. Hemorragia de vías \\ digestivas altas por presentación atípica de \\ adenocarcinoma endometrial metastásico \\ en el estómago. Tipificación diagnóstica \\ con recursos endoscópicos. Rev Colomb \\ Gastroenterol. 2021;36(Supl.1):42-46. https://doi. \\ org/10.22516/25007440.520 \\ Médico, cirujano gastrointestinal y \\ endoscópico. Líder del servicio de \\ gastroenterología y cirugía digestiva, Clínica \\ Foscal. Floridablanca, Santander, Colombia. \\ Médico interno, miembro del servicio de \\ gastroenterología y cirugía digestiva, Clínica \\ Foscal. Floridablanca, Santander, Colombia. \\ Médico, miembro, gastroenterólogo y \\ endoscopista, Clínica Foscal. Floridablanca, \\ Santander, Colombia. \\ Médico, miembro, patóloga. Servicio de \\ patología, Clínica Foscal, Floridablanca, \\ Santander, Colombia. \\ 5 Nutricionista, Clínica Foscal. Floridablanca, \\ Santander, Colombia. \\ *Correspondencia: Gloria Mendoza. \\ gliliana.9423@gmail.com \\ Fecha recibido: $\quad 05 / 03 / 20$ \\ Fecha aceptado: $27 / 05 / 20$

\section{(c)}

\section{Resumen}

El cáncer de endometrio es la neoplasia ginecológica maligna más frecuente en el sexo femenino. Su presentación metastásica se limita principalmente a los órganos vecinos y ganglios cercanos, y es infrecuente la invasión al tubo digestivo superior. En el presente artículo se presenta una paciente que ingresó al servicio de urgencias con cuadro de hemorragia de vías digestivas altas, enfocado inicialmente como un tumor estromal gastrointestinal (GIST) de la pared gástrica, pero que luego de la realización de una adecuada historia clínica, complementada con imágenes diagnósticas y avances ecoendoscópicos, se logró el diagnóstico de metástasis de cáncer de endometrio.

\section{Palabras clave}

Cáncer de endometrio, tracto gastrointestinal, hemorragia de las vías digestivas altas, metástasis.

\section{Abstract}

Endometrial cancer is the most common gynecologic malignancy in women. Its metastatic presentation is mainly limited to neighboring organs and nearby lymph nodes, and infiltration of the upper digestive tract is uncommon. This case report depicts a patient admitted to the emergency department with upper gastrointestinal bleeding symptoms. Initially, a gastrointestinal stromal tumor (GIST) of the gastric wall was suspected, but endometrial cancer metastasis was discovered after a thorough medical examination, diagnostic imaging, and echoendoscopes.

\section{Keywords}

Endometrial cancer; gastrointestinal tract; upper gastrointestinal bleeding; metastases.

\section{INTRODUCCIÓN}

La hemorragia gastrointestinal es considerada como una entidad clínica frecuente y la principal emergencia gastroen- terológica; corresponde al $83 \%$ de las hemorragias altas y al $17 \%$ de las bajas (1). Por una parte, las características actuales de los pacientes que pueden llegar a presentar hemorragias de vías digestivas altas han cambiado: presentan una 
mayor edad y tienen una comorbilidad altamente letal (2). Por otra parte, el cáncer de endometrio es la neoplasia ginecológica maligna más frecuente en países desarrollados; en su presentación histológica, es el adenocarcinoma de tipo endometroide (3). En Estados Unidos se documentan cerca de 60000 nuevos casos al año y aproximadamente 10000 muertes por año (4). En Colombia, según datos del GLOBOCAN de 2012, la incidencia ajustada por edad es de 3,6 cada 100000 mujeres/año (5). La presentación de cáncer de endometrio con metástasis en el tubo digestivo es infrecuente, y la afectación en el recto es la más frecuente por invasión local (6).

Se expone un caso infrecuente de cáncer de endometrio con metástasis al estómago, el cual tuvo una presentación clínica de hemorragia de vías digestivas altas de origen no claro. Fue registrada inicialmente con un tumor tipo GIST (7), pero posteriormente se documentó en el reporte de patología la presencia de tejido endometrial dependiente de la cuarta ecocapa de la pared gástrica.

\section{REPORTE DE CASO}

Se trata de una mujer de 71 años que ingresó al servicio de urgencias por un cuadro clínico de 18 horas de evolución consistente en dolor abdominal en el mesogastrio irradiado a la región lumbar asociado con deposiciones melénicas sin otra sintomatología asociada. Como antecedentes de importancia sufría de hipotiroidismo, era portadora de válvula biológica y con historia de cáncer de endometrio, el cual fue manejado en julio de 2012 con histerectomía total.

El reporte de patología evidenciaba el útero con adenocarcinoma endometrial tipo endometroide pobremente diferenciado, infiltrante hasta el $95 \%$ del espesor del miometrio, con 18 ganglios resecados libres de metástasis y citología de líquido peritoneal negativo para células malignas. En su momento se estadificó en grado I por un T1bN0M0 de alto riesgo, por lo que se decidió darle un tratamiento adyuvante con radioterapia externa y braquiterapia. La paciente abandonó el tratamiento luego de la primera sesión y no realizó la braquiterapia complementaria. Debido a que se enfocó a la paciente con un cuadro de hemorragia de vías digestivas altas, se realizó una endoscopia con evidencia de lesión subepitelial de 4 x 4 $\mathrm{cm}$ ulcerada en la unión antrocorporal-curvatura mayor. El cuadro hemático resultó sin leucocitosis, sin neutrofilia, sin anemia, sin trombocitopenia y con tiempos de coagulación dentro de límites normales.

Se consideró inicialmente que la paciente se encuentra cursando con GIST gástrico ulcerado en el cuerpo gástrico distal sin sangrado activo y se complementó el estudio para evaluar el compromiso locorregional con la realización de tomografía axial computarizada (TAC) de abdomen y pel- vis con hallazgos de masa del estroma gastrointestinal en el espacio gastrocólico compatible con un tumor de GIST (Figura 1) y masa o adenomegalia retroperitoneal por detrás de la cabeza de páncreas (Figura 2).

Ante el hallazgo de masa o adenomegalia retroperitoneal, las cuales no son presentaciones usuales de un tumor estromal tipo GIST, se realizó una ultrasonografía endoscópica (8) gástrica con el equipo radial Pentax-Noblux y en la visión endoscópica se identificó una lesión subepitelial en la curvatura mayor de la unión antrocorporal; en la visión ecográfica se trata de una lesión hiperecogénica de 40 × 38 $\mathrm{mm}$ de bordes bien definidos sin calcificaciones ni necrosis, que compromete toda la pared del estómago (Figura 3).

Posteriormente, se realizó una elastografía cualitativa (9) de color azul-verde y cuantitativa strain ratio de 10 y strain histograma del $95 \%$. En la ventana duodenal se identificó una adenopatía de 40 × $45 \mathrm{~mm}$ retropancreática hiperecogénica de bordes bien definidos (Figura 4). A continuación, se documentó una elastografía de color azul al realizar elastografía cualitativa, strain ratio de 10 y strain histograma del $44 \%$ (Figura 5).

Con la correcta caracterización de la lesión, se decidió utilizar un ecoendoscopio lineal para efectuar la punción de la

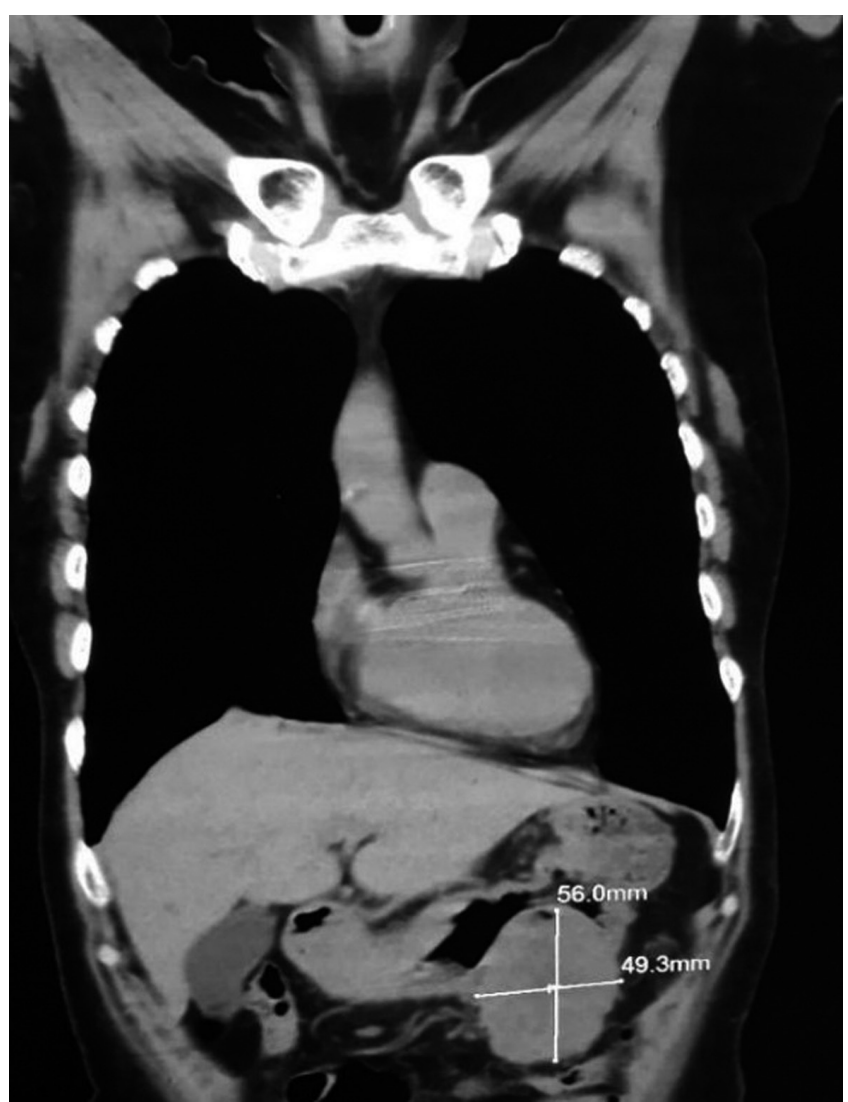

Figura 1. TAC de la masa en la pared gástrica. 


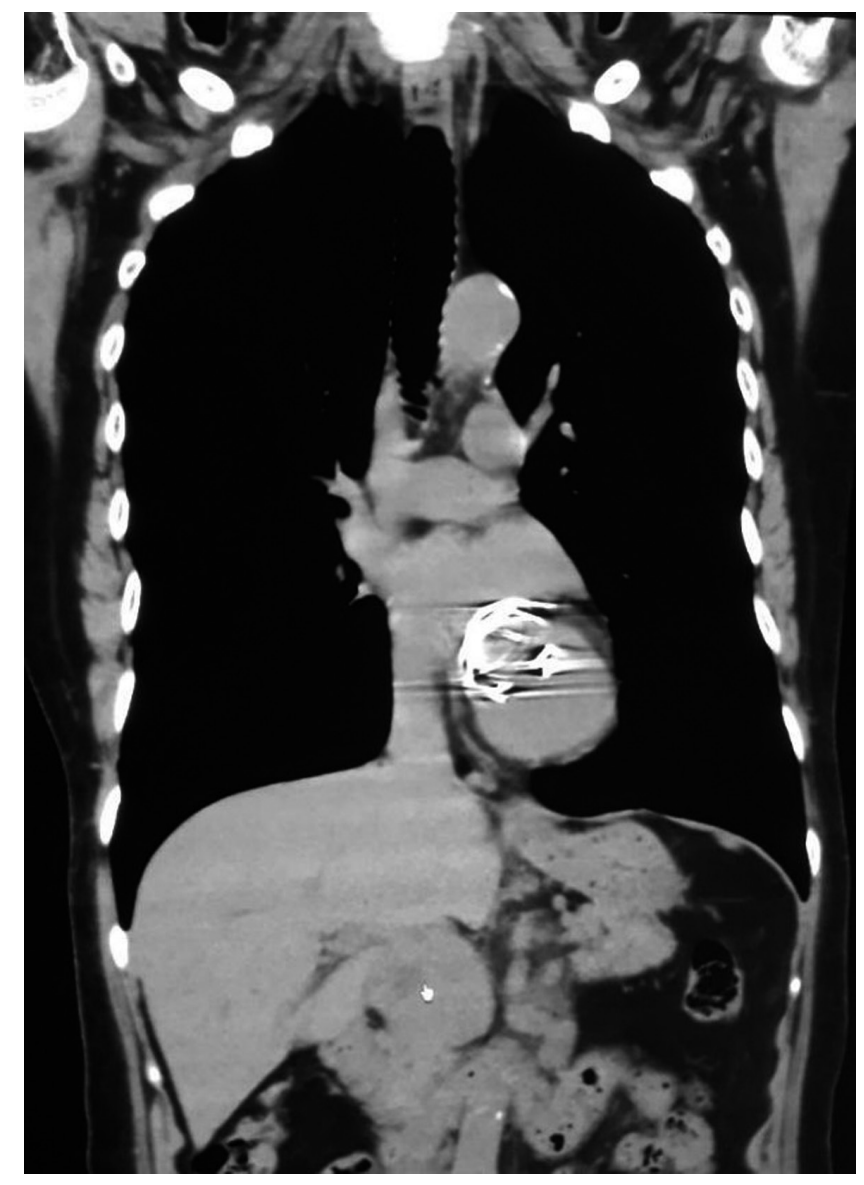

Figura 2. TAC de la masa o adenomegalia retroperitoneal detrás de la cabeza de páncreas.

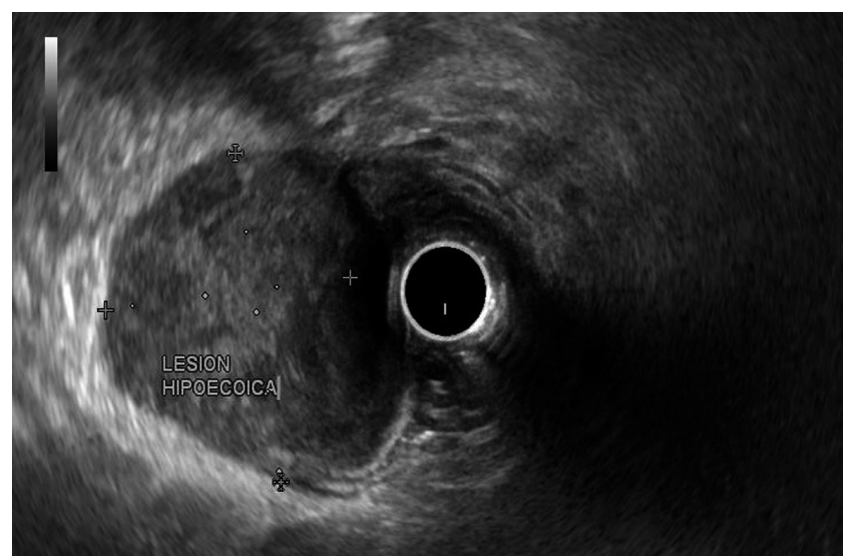

Figura 3. Lesión hiperecogénica gástrica.

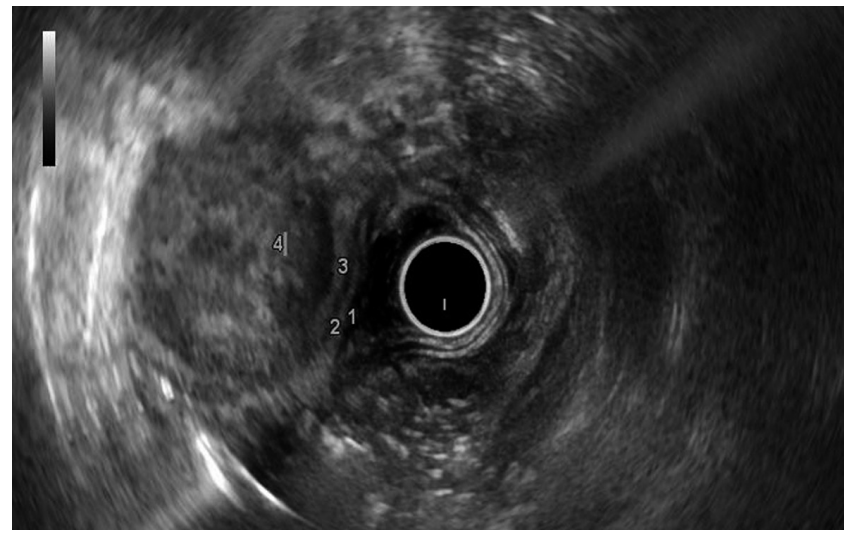

Figura 4. Lesión retropancreática.

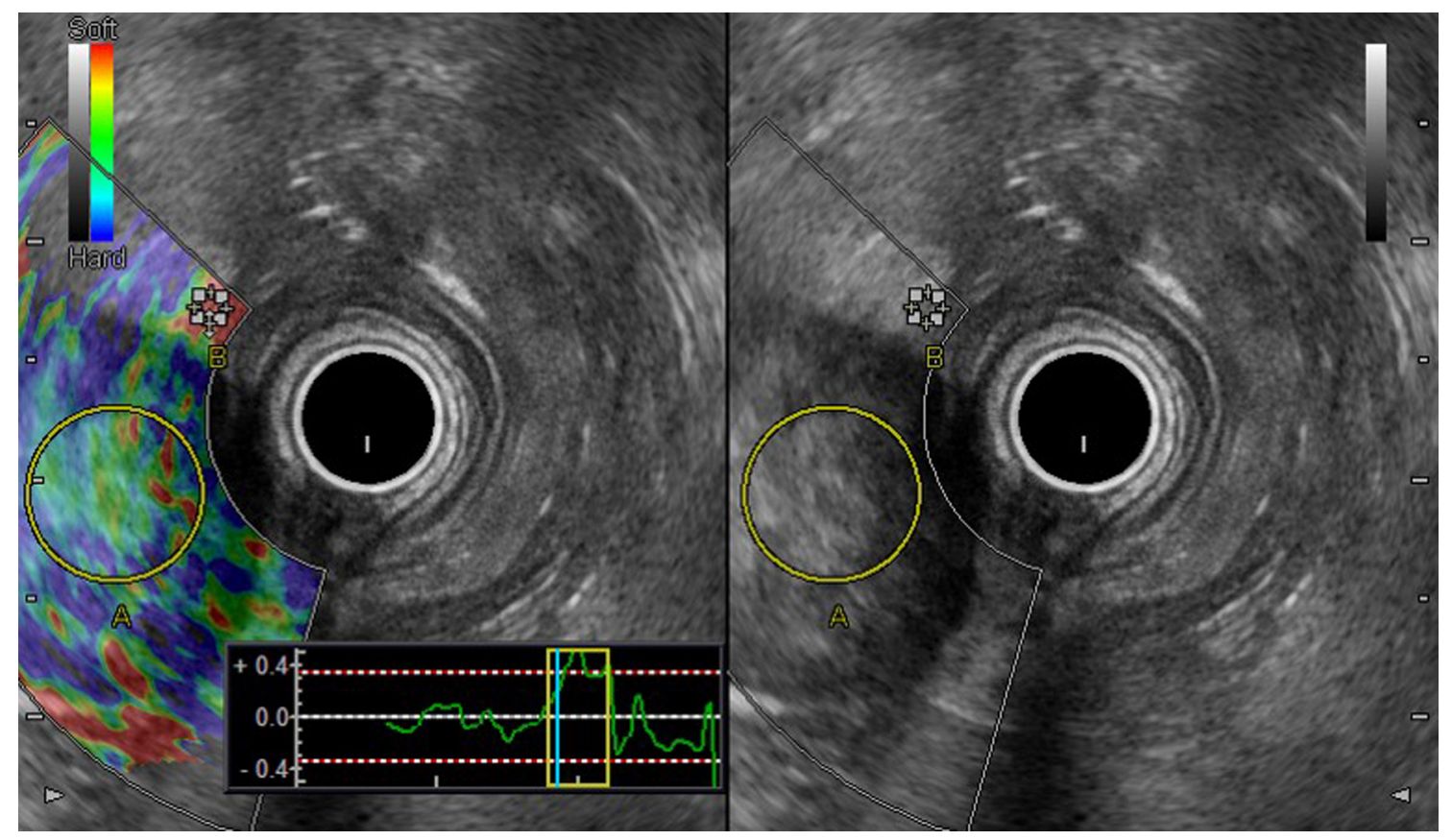

Figura 5. Elastografía cualitativa. 
adenopatía retropancreática con una aguja n. ${ }^{\circ} 22$ (Figura 6). Se obtuvo una muestra y se envió a estudio histológico e inmunohistoquímico y se realizó otra punción en la lesión gástrica subepitelial descrita para el mismo estudio. En el reporte de patología de la lesión del estómago y la adenopatía retropancreática se obtuvieron los siguientes hallazgos: carcinoma pobremente diferenciado de origen ginecológico e inmunofenotipo de adenocarcinoma endometroide pobremente diferenciado (Figura 7). Con estos hallazgos se inició el manejo médico a cargo del servicio de oncología, el cual indicó el tratamiento con carboplatino más paclitaxel.

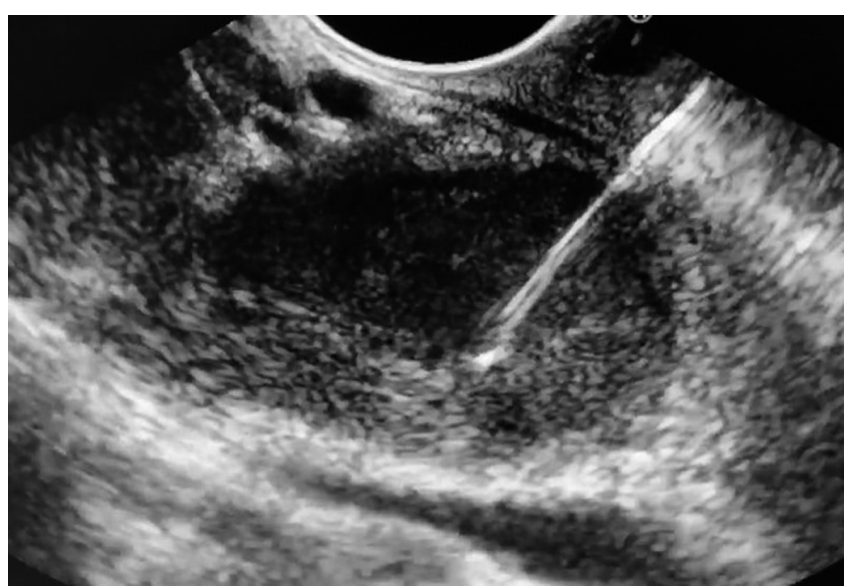

Figura 6. Punción de la lesión retropancreática con aguja n. ${ }^{\circ} 22$. $^{2}$

\section{DISCUSIÓN}

El cáncer de endometrio es la neoplasia maligna más frecuente de origen ginecológico, y dentro de los cánceres de origen femenino está en el $6 \%$ de estos (10). La presentación metastásica en los estadios más avanzados de este tipo de cáncer es, en mayor frecuencia, en los órganos cercanos, los cuales se encuentran ubicados en la pelvis, el peritoneo y los ganglios linfáticos pélvicos, paraaórticos e intraabdominales (11). La presentación en el tubo digestivo es realmente infrecuente, y el recto es la porción más afectada por este cáncer (13). La importancia de este caso es su presentación en la pared gástrica, que compromete toda la pared del estómago, lo que inicialmente generó una confusión en su enfoque diagnóstico debido a su presentación clínica como hemorragia de vías digestivas altas y por su distribución macroscópica que se asemejaba con un tumor tipo GIST.

Adicionalmente, por una adecuada anamnesis con esclarecimiento de los antecedentes ginecológicos de la paciente, la ayuda complementaria de las imágenes diagnósticas como el uso del TAC abdominopélvica para evaluar el compromiso, la adecuada visualización ecoendoscópica de las lesiones gástrica y retropancreática de las que se tomó biopsia, y un posterior estudio histológico con una caracterización satisfactoria de la etiología, finalmente, con la suma de los hallazgos y el trabajo multidisciplinario, se cambió totalmente el abordaje terapéutico de la lesión, que inicialmente sería quirúrgico, a un manejo completamente médico con quimioterapia de rescate por parte del servicio de oncología.

\section{CONCLUSIÓN}

La importancia de este caso es dar a conocer una de las formas infrecuentes de presentación de metástasis de cáncer de endometrio y el modo en que una presentación clínica
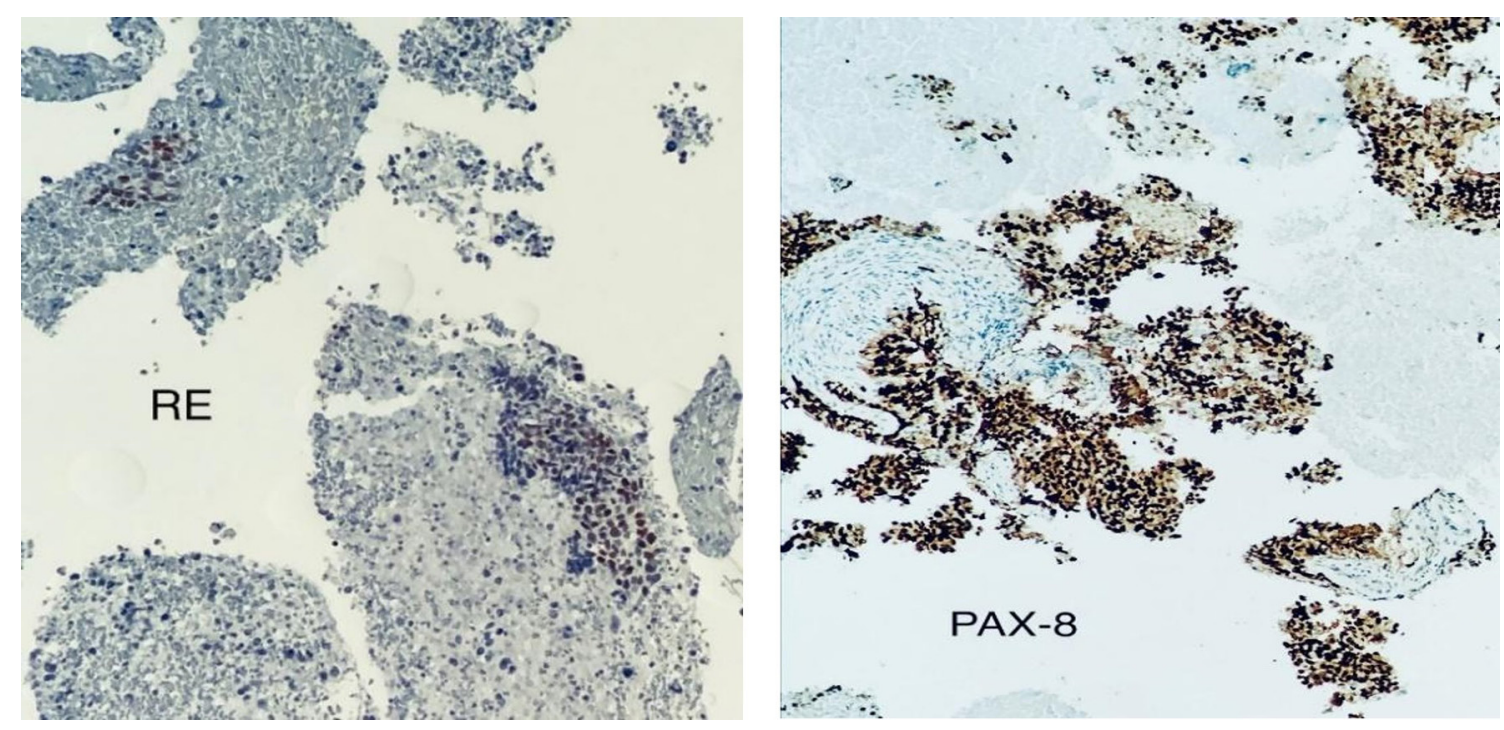

Figura 7. Muestra del reporte de patología. 
de hemorragia de vías digestivas altas, con las características descritas, puede desorientar la impresión diagnóstica inicial. No obstante, con la ayuda de los nuevos avances tecnológicos de la cirugía gastrointestinal y ecoendoscópica, se pueden tomar muestras de tejidos con alta precisión para una adecuada identificación de lesiones, de modo que se pueden cambiar las conductas terapéuticas para darle la mejor opción de tratamiento a los pacientes.

\section{REFERENCIAS}

1. Ichiyanagui Rodriguez C. Epidemiología de la hemorragia digestiva. Act Med Per. 2006;23(3):152-5.

2. Lambert R, Hainaut P. The multidisciplinary management of gastrointestinal cancer. Epidemiology of oesophagogastric cancer. Best Pract Res Clin Gastroenterol. 2007;21(6):921-45. https://doi.org/10.1016/j. bpg.2007.10.001

3. Chen L, Bereck J. Endometrial carcinoma: Clinical features, diagnosis, prognosis and screnning [internet]. UpToDate; 2019 [citado el 28 de enero de 2020]. Disponible en: https://www.uptodate.com/contents/endometrial-carcinoma-clinical-features-diagnosis-prognosis-and-screening

4. Miller KD, Nogueira L, Mariotto AB, Rowland JH, Yabroff KR, Alfano CM, Jemal A, Kramer JL, Siegel RL. Cancer treatment and survivorship statistics, 2019. CA Cancer J Clin. 2019;69(5):363-385. https://doi.org/10.3322/ caac. 21565

5. Global Cancer Observatory [Internet]. Lyon: IARC; 2012 [citado el 28 de enero de 2020]. Disponible en: https:// gco.iarc.fr/

6. Ibáñez Pinto A, Fernández Salgado E, Castro Ortiz E, Baltar Arias R, Vázquez Vázquez S, Ledo Barro L, Vázquez San Luis J, Vázquez Astray E. Hemorragia digestiva de origen incierto por un adenocarcinoma endome- trial metastásico. Respuesta al tratamiento hormonal. Gastroenterol Hepatol. 2007;30(9):530-4. https://doi. org/10.1157/13111689

7. Oyanedel R, O’brien A, Pizarro A, Zamora E, Menias C. Tumor Estromal gastrointestinal (GIST): formas de presentación. Rev Chil Radiol. 2005;11(1):13-8. https://doi. org/10.4067/S0717-93082005000100005

8. Abu Dayyeh BK, Levy MJ. Therapeutic endoscopic ultrasound. Gastroenterol Hepatol (N Y). 2012;8(7):450-6.

9. Guzmán Aroca F, Abellán Rivera D, Reus Pintado M. La elastografía: una nueva aplicación de la ecografía. ¿Cuál es su utilidad clínica? Radiologia. 2014;56(4):290-4. https://doi.org/10.1016/j.rx.2012.09.006

10. Jemal A, Siegel R, Ward E, Murray T, Xu J, Smigal C, Thun MJ. Cancer statistics, 2006. CA Cancer J Clin. 2006;56(2):106-30. https://doi.org/10.3322/canjclin.56.2.106

11. Alvarado-cabrero I. Adenocarcinoma de endometrio: Conceptos actuales. Gac Mex Oncol. 2012;11(3):196-202.

12. Alvarez Sánchez JA, Fernández Lobato R, Aramburo González JA, Fradejas López JM, Marín Lucas J, Moreno Azcoita M. Infiltración rectal por adenocarcinoma tubárico endometroide como causa de rectorragia. Rev Esp Enferm Dig. 1995;87(7):549-50. 